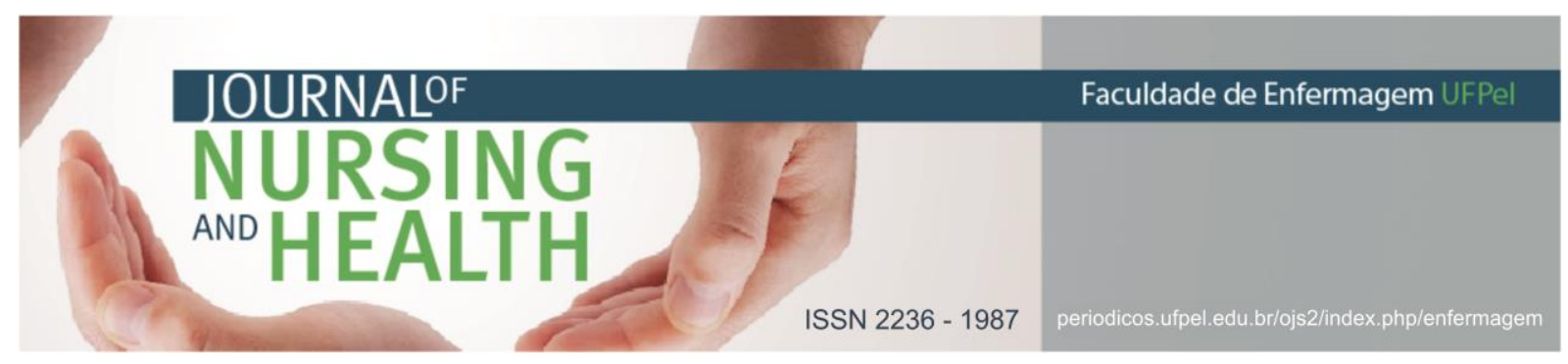

ARTIGO ORIGINAL

\title{
Estratégias de atendimento à crise psíquica por um serviço de atendimento móvel de urgência
}

\author{
Strategies for dealing with the psychic crisis by an emergency mobile service \\ Estrategias de atención a la crisis psíquica por un servicio de atención móvil de \\ urgencia
}

Souza, Alana dos Santos de ${ }^{1}$; Pinho, Paula Hayasi²; Vera, Sinara ${ }^{3}$; Cortes, Helena Moraes ${ }^{4}$

Como citar este artigo: Souza AS, Pinho PH, Vera S, Cortes HM. Estratégias de atendimento à crise psíquica por um serviço de atendimento móvel de urgência. J. nurs. health. 2019;9(1):e199109

\section{RESUMO}

Objetivo: conhecer quais estratégias estão sendo adotadas no atendimento à crise psíquica pelos profissionais de um Serviço de Atendimento Móvel de Urgência num município do Recôncavo da Bahia. Métodos: estudo qualitativo, descritivo e exploratório. Foram participantes, 22 profissionais que prestam assistência direta aos usuários do serviço. Coletaram-se os dados por meio de entrevista semiestruturada em abril de 2018, analisou-se os dados por meio da técnica de análise temática. Resultados: evidenciou-se como estratégias de atendimento: a avaliação da cena, ação conjunta com a polícia militar, ações de contenção física e química, orientação à família, encaminhamento do usuário para o serviço hospitalar. Considerações finais: as estratégias de atendimentos a crise psíquica são fundamentadas na psiquiatria clássica, havendo a necessidade de educação permanente das equipes do referido serviço, baseando-se nos cuidados fundamentados no modo de atenção psicossocial.

Descritores: Intervenção na crise; Saúde mental; Serviços de saúde mental; Psiquiatria; Transtornos mentais.

\begin{abstract}
Objective: to know what strategies are being adopted in the care of the psychic crisis by the professionals of emergency mobile service in a municipality of the Recôncavo of Bahia. Methods: this is a qualitative, descriptive and exploratory study. Twenty-two professionals who provide direct assistance to the users of the service were participants of this study. The data were collected by means of a semistructured interview in April of 2018 and was used the technique of content thematic analysis. Results: the evaluation of the scene, joint action with the military police, actions of physical and chemical containment, orientation to the family, and referral of the user to the hospital service were evidenced. Final considerations: the strategies for attending to the psychic crisis are based on the classic psychiatric way, there is a need for permanent education of the emergency mobile service teams based on the psychosocial care.
\end{abstract}

Descriptors: Crisis intervention; Mental health; Mental health services; Psychiatry; Mental disorders.

1 Enfermeira. Universidade Federal do Recôncavo da Bahia (UFRB). E-mail: alanasanttos94@gmail.com http: / / orcid.org/0000-0002-8252-1795

2 Psicóloga. Doutora em Ciências. Universidade Federal do Recôncavo da Bahia (UFRB). E-mail: phpinho@ufrb.edu.br http://orcid.org/0000-0001-8922-0699

3 Enfermeira. Mestre em Desenvolvimento Regional e Meio Ambiente. Universidade Federal do Recôncavo da Bahia (UFRB). E-mail: sivera1@hotmail.com http://orcid.org/0000-0001-5263-0301

4 Enfermeira. Doutora em Ciências. Universidade Federal do Recôncavo da Bahia (UFRB). E-mail: helena@ufrb.edu.br http://orcid.org/0000-0001-8538-8400 


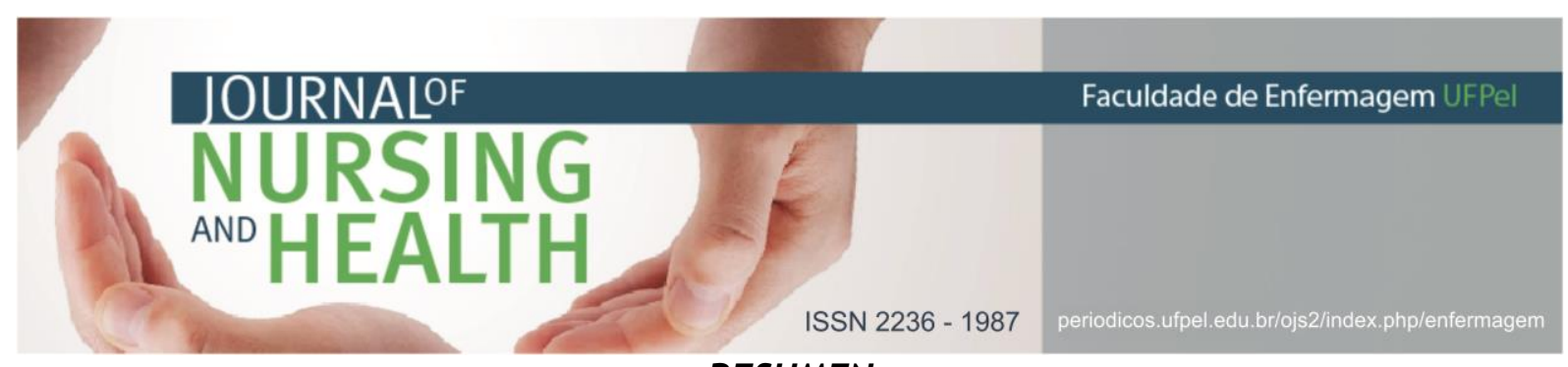

RESUMEN

Objetivo: conocer qué estrategias están siendo adoptadas en la atención a la crisis psíquica por los profesionales del servicio de atención móvil de urgencia en un municipio del recôncavo baiano. Métodos: estudio cualitativo, descriptivo y exploratorio. Se estudiaron 22 profesionales que prestan asistencia directa a los usuarios del servicio. Se recogen los datos por medio de una entrevista semiestructurada en abril de 2018 y se analizaron los datos por medio de la técnica de análisis temático. Resultados: se evidenció como estrategias: la evaluación de la escena, acción conjunta con la policía militar, acciones de contención física y química, orientación a la familia, encaminamiento del usuario para el servicio hospitalario. Consideraciones finales: las estrategias de atención a la crisis psíquica se fundamentan en la psiquiatría clásica, hay necesidad de educación permanente de los equipos, basados en los cuidados de la atención psicosocial.

Descriptores: Intervención en la crisis (Psiquiatría); Salud mental; Servicios de salud mental; Psiquiatría; Trastornos mentales.

\section{INTRODUÇÃO}

A crise psíquica situa-se no âmbito das urgências ou emergências psiquiátricas, embora nem toda situação de crise em saúde mental, caracteriza-se por uma emergência. ${ }^{1}$ Nesta condição, essa pode ser compreendida e conceituada pelas seguintes formas: os surtos psicóticos, as tentativas de suicídios, as depressões, as síndromes cerebrais orgânicas, as alterações do comportamento devido ao uso abusivo de álcool e outras drogas, entre outras. $^{2}$

O modelo clássico da psiquiatria tradicional compreende a crise como uma situação de grave desequilíbrio em decorrência da doença mental. 0 saber da psiquiatria clássica conserva e prioriza ações que visam o controle do indivíduo, por meio de medidas de contenção, isolamento e redução dos sintomas, cujas práticas eram (são) frequentemente utilizadas no cotidiano dos manicômios. ${ }^{3}$

No campo da saúde mental, a crise é compreendida como sofrimento psíquico intenso, causando uma desorganização mental, em detrimento das condições vivenciadas no contexto familiar e social do sujeito, requerendo dos serviços de saúde, acolhimento, responsabilização e construção de vínculo entre profissionais e a pessoa em sofrimento psíquico. $^{3}$

Neste contexto, a crise não se limita à agudização sintomatológica, à exacerbação de sintomas e à perturbação da ordem social. ${ }^{4}$ Trata-se de um momento de conflito psíquico marcado pelo medo, dor e incerteza, e é expresso a partir das singularidades de cada pessoa. ${ }^{5}$ Esta compreensão deve guiar toda assistência de saúde mental. 4

As discussões sobre a violação dos direitos humanos e cidadania, acerca do modelo biomédico frente a assistência à saúde mental vigente por muitos anos, contribuíram para que fossem instituídas novas estratégias políticas, científicas e sociais que visassem a valorização do cuidado ao sujeito, garantindo sua autonomia e sua independência. ${ }^{4}$ Uma importante estratégia foi a criação da Rede de Atenção Psicossocial (RAPS), ganho do movimento da Reforma Psiquiátrica 


\section{IOURNALOF

surgido no final da década de 1970 até atualidade. ${ }^{4}$

A Reforma Psiquiátrica redirecionou a assistência à saúde mental, na perspectiva de substituir o modelo manicomial e hospitalocêntrico por uma ampla e diversificada rede de serviços de base territorial. ${ }^{6}$ Nesta perspectiva, o atendimento à crise psíquica passou a ser realizado pelos seguintes pontos de atenção: Serviço de Atendimento Móvel de Urgência (SAMU), Centros de Atenção Psicossocial (CAPS) nas suas diferentes modalidades, emergências dos hospitais gerais, Portas Hospitalares de Atenção às Urgências (Pronto Socorro), Unidade de Pronto Atendimento (UPA) e pela Atenção Básica. $^{7}$

Contudo, apesar da ampliação de acesso dos serviços à saúde mental, o Ministério da Saúde preconiza que, o atendimento à crise deve acontecer especialmente no lugar onde o sujeito em sofrimento psíquico encontra-se, ou seja, nos locais do seu contexto de vida ou de cuidado: residência, via pública, serviços de saúde comunitários, como o CAPS e a atenção básica, dentre outros. ${ }^{8}$

Dessa forma, os serviços de urgência e emergência como o SAMU assumem um lugar importante na atenção às pessoas em situação de crise, compreendendo a assistência pré-hospitalar, cujo atendimento é prestado nos locais onde a pessoa em sofrimento psíquico encontra-se. ${ }^{9}$ Assim, ressalta-se a importância do SAMU na RAPS. Em virtude, de ser porta de entrada para os usuários que possuem transtornos mentais, em situação de crise psíquica, no Sistema
Único de Saúde (SUS), tem como responsabilidade 0 cuidado com as urgências e/ou emergências em saúde mental. ${ }^{1}$

A articulação do SAMU com a regulação das urgências em saúde mental é essencial para o fortalecimento e a ampliação das ações de saúde mental no SUS, pois oferecem atendimento inicial aos usuários em situação de crise no seu território e, encaminhamentos para outros serviços da rede. É a porta de entrada itinerante, fazendo a articulação da demanda de saúde mental até outros pontos da RAPS. ${ }^{10}$ Em muitos países, os usuários em situações de crise psíquica, também são encaminhados por algum tipo de serviço móvel de saúde mental e urgência. $^{11}$

0 movimento da Reforma Psiquiátrica, também traz mudanças propostas na atenção à saúde mental, na qual implica que o cuidado ao sujeito em sofrimento psíquico deve ser permeado por práticas e posturas de respeito, ética e coresponsabilidade. ${ }^{3}$ Entretanto, estudos tem mostrado que a visão estigmatizada, e o medo excessivo que os profissionais de saúde possam nutrir frente à pessoa com transtornos mentais acabam por alimentar o emprego das práticas coercitivas durante as intervenções na crise psíquica, indo de encontro do que é proposto pela Reforma Psiquiátrica brasileira e pelo modo de atenção psicossocial. ${ }^{1}$

Muitos profissionais de saúde ainda parecem preservar um pensamento manicomial, $e$ isto interfere diretamente no cuidado às 


\section{IOURNALF

pessoas com transtornos mentais e na própria compreensão de uma RAPS efetiva, ${ }^{12}$ impactando negativamente na assistência direta aos usuários de saúde mental, no contexto de serviços como o SAMU. ${ }^{1}$ A garantia de uma sociedade livre do modelo manicomial, não se firma apenas na ausência dos hospitais psiquiátricos, pois esta mesma sociedade pode continuar fortemente manicomial, de acordo com as práticas adotadas na atenção a crise pelos profissionais de saúde, por exemplo. ${ }^{13}$

Dessa forma, tornou-se premente problematizar e discutir as práticas adotadas pelos profissionais de saúde no atendimento à crise psíquica, a fim de que se possa contribuir para concretizar o processo da Reforma Psiquiátrica brasileira nos serviços de urgência e emergência como o SAMU, no contexto do recôncavo baiano. Assim, emerge 0 seguinte questionamento: quais as estratégias estão sendo utilizadas pelos profissionais que atuam no SAMU durante $o$ atendimento à crise psíquica num município localizado no recôncavo baiano? Este trabalho teve por objetivo conhecer quais estratégias estão sendo adotadas no atendimento à crise psíquica pelos profissionais de um Serviço de Atendimento Móvel de Urgência num município do Recôncavo da Bahia.

\section{MATERIAIS E METÓDOS}

Trata-se de um estudo qualitativo, descritivo e exploratório. A presente pesquisa foi realizada em um Serviço de Atendimento Móvel de Urgência (SAMU 192), de um município do Recôncavo da Bahia. O município, palco deste estudo, dispõe apenas de um SAMU composto por cinco equipes, que faz a cobertura dos atendimentos da cidade e dos municípios do entorno. Este serviço contava com uma ambulância - configurada como Unidade de Suporte Avançado de vida (USA) - composta por condutor socorrista, médico e enfermeiro; e outra ambulância configurada como Unidade de Suporte Básico de vida (USB), composta por condutor socorrista e técnico de enfermagem.

0 critério de inclusão dos participantes do estudo foi serem trabalhadores do SAMU que atuam diretamente na prestação da assistência aos usuários do serviço no território. O serviço era composto por 29 profissionais, sendo entrevistados cinco médicos, três enfermeiros, sete técnicos de enfermagem e sete condutores socorristas, perfazendo um total de 22 profissionais entrevistados. Não participaram sete profissionais, pois três não compareceram ao serviço no período da coleta de dados, dois estavam em gozo de férias e dois recusaram-se a participar.

Os participantes foram abordados e convidados a participar da pesquisa no seu local de trabalho, durante os turnos da manhã e da tarde, de acordo com sua disponibilidade. As entrevistas ocorreram em um prédio denominado como base do SAMU, após os profissionais serem informados dos objetivos do estudo e esclarecidos quanto os preceitos éticos. Foi solicitado a assinatura no Termo de Consentimento Livre e Esclarecido.

Utilizou-se uma entrevista semiestruturada para a coleta dos dados, a qual foi gravada 


\section{ISSN 2236 - 1987}

individualmente, com um tempo de duração média entre 30 a 40 minutos, realizada por uma das autoras deste estudo, a qual recebeu capacitação antes ir ao campo da pesquisa. 0 roteiro da entrevista foi constituído com variáveis sociodemográficas e questões que abordaram a assistência ao usuário no atendimento crise psíquica no cotidiano de trabalho dos profissionais do SAMU. Os dados foram coletados em abril de 2018.

Após a transcrição dos áudios das entrevistas, o material foi relido inúmeras vezes, procedimento definido como "leitura flutuante", que permite apreender as ideias centrais dos participantes da pesquisa sobre o tema em foco. ${ }^{14}$ Dessa forma, organizaram-se os relatos e classificaram-se os dados de acordo com a semelhança dos temas surgidos.

0 procedimento eleito para análise dos dados foi a técnica de análise de conteúdo temática, em que o conceito central é o tema, o qual está ligado a uma afirmação a respeito de determinado assunto. Esse comporta um feixe de relações e pode ser graficamente representado por meio de uma palavra, frase ou resumo sintético. ${ }^{14}$ Com a finalidade de garantir 0 anonimato dos participantes, os mesmos foram identificados pela ordem em que foram sendo entrevistados sequencialmente (ex.: M1, M2, E1, E2, C1, C2, TEC1, TEC2), obedecendo a seguinte legenda: M (Médicos), E (Enfermeiros), $C$ (Condutores socorristas), TEC (Técnicos de enfermagem), seguidos pelo número arábico que representa a ordem que os sujeitos foram sendo entrevistados. Esta pesquisa foi submetida ao Comitê de Ética e Pesquisa da Universidade Federal do Recôncavo da Bahia (CEP-UFRB) e aprovada sob o Certificado de Apresentação para Apreciação Ética: 82569818.9.0000.0056, sendo respeitados todos os princípios éticos e legais em todas as etapas da pesquisa, de acordo com a resolução $\mathrm{n}^{\circ}$ 466/2012, do Conselho Nacional de Saúde.

\section{RESULTADOS}

Participaram do estudo 22 profissionais, destes $77,27 \%$ eram do sexo masculino e $22,73 \%$ do sexo feminino, a idade média dos participantes foi de 40 anos e o tempo de atuação no SAMU foi de 5 a 6 anos. Da análise dos dados, emergiu uma categoria empírica denominada "atendimento às crises psíquicas: estratégias adotadas", subdividida em seis subcategorias, a saber: avaliação da cena, ação conjunta do SAMU com a polícia militar, assistência centrada na contenção química e física, orientação à família, encaminhamento das famílias e das pessoas em situação de rua para assistência social, e encaminhamento do usuário para outros pontos da RAPS.

\section{Atendimento às crises psíquicas: estratégias adotadas}

Avaliação da cena

0 entendimento de crise psíquica é caracterizado pelos profissionais do SAMU como ocorrências que podem oferecer risco para a equipe e para outros, em virtude disso, a concepção dos profissionais é de que as 


\section{JOURNALOF \\ NURSING \\ aNO HEALTH}

ISSN 2236 - 1987

Sempre vai com a polícia, sempre é com a polícia, porque um indivíduo é [...], que tem transtornos mentais, a qualquer momento ele pode se transformar, é o potencial perigo tanto para ele quanto para as pessoas que estão ao entorno, principalmente para a equipe. (M2)

A gente primeiro solicita $o$ suporte da PM né, porque a gente não sabe como é que está, como é que a gente encontra esse paciente, às vezes ele está agressivo, às vezes não, então assim, a gente precisa do apoio da PM para conter. (E1)

Geralmente, o paciente quando está nesse estado, é agressivo, a gente na maioria das vezes vai com a presença da polícia, vamos escoltados para que não haja nenhum tipo de agressão com a equipe, então a polícia faz essa proteção. (C6)

Entretanto, a ação conjunta da PM na atenção à crise pode prejudicar o atendimento e a relação dos profissionais com os usuários. A presença da viatura da PM e, até da própria sirene da ambulância, que se assemelha com a da polícia, são motivos para assustar o usuário, em virtude disso, os profissionais algumas vezes não conseguem realizar o atendimento, como ficam evidenciados nas frases temáticas a seguir:

[...] às vezes acontece de a gente chegar no atendimento $e$ o paciente evadindo, com a 


\section{.}

presença da polícia, da viatura e tudo mais, a gente acaba até voltando, às vezes sem prestar o atendimento por conta disso. (C6)

[...] às vezes a gente vai depender do paciente que tem uma boa parte de que já conhecemos quem é o paciente, $a$ galera já sabe, a gente já vai e deixa a ambulância distante $e$ desliga a sirene, até porque para não chamar a atenção, e achar que é a polícia. (C3)

0 atendimento ao usuário em crise, em situação de rua, também é realizado em conjunto com a PM, conforme as seguintes frases temáticas:

[...] o paciente na rua a polícia chega [...] alguém pega $e$ contenha o paciente caso contrário diretamente não é nossa função fazer, não é nem porque a gente não quer, é porque não somos treinados para aquilo entendeu. (C3)

O sem família e está agressivo na rua é o policial que vem ajudar, a gente contem, a gente faz o processo. (C7)

\section{Assistência centrada contenção física e química}

na

As intervenções citadas pelos profissionais do SAMU ao usuário em crise, são baseadas na contenção física e química; durante o atendimento à crise, a PM realiza a contenção física e - SAMU a contenção mecânica, associando-se à intervenção
ISSN 2236 - 1987

medicamentosa,

como

fica evidenciado nas falas a seguir:

[...] quando a gente vai para uma situação dessas, para um paciente psiquiátrico a gente já vai mesmo para tentar medicar $e$ trazer. (TEC1)

[...] geralmente precisa conter, na maioria das vezes precisa conter, e fazer uma medicação intramuscular e conduzir. (E1)

E sempre com ajuda da polícia militar se for uma crise que ele esteja muito agressivo, a gente nunca encosta no paciente, sem uma ajuda da polícia militar para poder fazer a contenção primeiro. (M5)

Porém, os profissionais do SAMU relatam que a contenção física feita pela PM muita das vezes, é compreendida de forma negativa pelos familiares:

A questão da agressividade, é o seguinte os familiares têm uns que libera você segurar para fazer uma contenção, uma imobilização, até para aplicar uma medicação, já tem familiares que não deixam né, tem essa questão. (C1)

[...] muitas vezes nem o policial quer segurar porque depois a família fica dizendo - "ah agrediu aquele negócio todo...”. (TEC1)

Tem família que fica assustada, às vezes acha que a gente tá machucando porque se precisa fazer uma contenção e, aí vai um profissional para cada lado 
tentando deitar na prancha, fazer uma contenção, amarrar na prancha $e$, aí às vezes tem familiar que não sabe e acha que a gente tá machucando, mas na verdade $a$ gente não tá machucando, a gente tá preservando tanto uma lesão física do paciente quanto uma lesão física para a gente e para o familiar. (E3)

\section{Orientação à família}

No atendimento à crise, os profissionais realizam orientação à família sobre a intervenção medicamentosa e a necessidade do encaminhamento do usuário para a rede hospitalar.

Você primeiro, precisa mostrar para família que você vai usar uma medicação para sedá-lo, e essa medicação pode prolongar um pouquinho mais, e o paciente ficar adormecido por um período prolongado, daí a necessidade de ser transferido para unidade hospitalar. (M1)

Aqui no SAMU nós acabamos orientado apenas a família como o SUS funciona, e deixando no hospital [...]. (M3)

Encaminhamento da família e das pessoas em situação de rua para a assistência social

Os profissionais do SAMU encaminham os familiares do usuário em crise, e as pessoas em situação de rua, para o serviço social do ponto da RAPS para onde foi levado o usuário.
Pacientes em situação de rua que tem uma crise e alguém liga, mas não tem ninguém para vim com esse paciente para o hospital, só que a gente não pode negar o socorro a esse tipo de paciente, aí a gente faz o atendimento, remove até o hospital e ao chegar no hospital esse paciente passa pela triagem e a gente vai direto no serviço social. (TEC4)

[...] é [...] seja ele levado para o CAPS tipo III, ou hospital psiquiátrico, ou hospital de referência, chegando lá a gente passa o caso para assistente social. (M1)

\section{Encaminhamento do usuário para outros pontos da RAPS}

O encaminhamento do usuário em crise para outros serviços da RAPS depende da conduta do médico regulador, que vai definir para onde encaminhar o usuário. Foi evidenciado pelos profissionais do SAMU que na maioria dos atendimentos à crise, o encaminhamento dá-se para o hospital geral da região, nos casos em que os usuários apresentem agressividade e/ou necessidade de contenção física ou química.

\section{[...] em surto geralmente encaminho para o hospital [geral]. (M4)}

99\% dos casos encaminha para o hospital [...] algumas vezes o próprio CAPS acolhe, mas quando ele está muito agressivo, aí a gente tem que trazer para o hospital geral. (C3) 


\section{JOURNALOF \\ NURSING \\ ANO HEALTH}

ISSN 2236 - 1987

0 bom seria se tivesse um CAPS $24 \mathrm{~h}$ ou um CAPS final de semana [...] o bom seria um CAPS III. (C4)

\section{DISCUSSÃO}

Em relação ao atendimento às urgências e emergências, no campo da saúde mental, a avaliação da cena baseia-se em realizar a observação e o reconhecimento das características relativas às particularidades de cada situação. Consiste, pois na leitura dos diversos aspectos que atuam no contexto de vida do sujeito, envolvendo a avaliação do ambiente social do usuário, o seu domicílio e o seu território, os conflitos na sua rede social e familiar, o nível de consciência e a sua subjetividade, os aspectos envolvidos a riscos de suicídios e de violência, indícios de dependência e/ou uso abusivo de álcool e outras drogas. ${ }^{10}$

O protocolo nacional do SAMU, apresenta a avaliação da cena como uma das condutas no manejo ao usuário em crise em saúde mental. ${ }^{15}$ Percebe-se que a avaliação da cena nos atendimentos de crise psíquica, pode ser uma excelente estratégia para os profissionais de saúde, entretanto, observa-se na fala dos sujeitos entrevistados que, esta avaliação é voltada para a segurança da equipe, e não para as necessidades que o usuário em sofrimento psíquico apresenta.

0 entendimento de crise psíquica pelos profissionais do serviço estudado está associado à periculosidade, no qual a equipe deve se proteger durante o atendimento, diante disso, é possível identificar nas falas dos sujeitos, ações e concepções vinculadas ao senso comum de que o indivíduo que possui 
transtorno mental é perigoso. Defender a ideia de que todo usuário dos serviços de saúde mental em crise é eventualmente agressivo e perigoso, demonstra preconceito e falta de capacitação dos profissionais de saúde para abordar e lidar com os atendimentos de crise. ${ }^{1}$

Os profissionais do SAMU adotam uma postura de defesa por entenderem que poderão ser agredidos, assim, para lidar com os atendimentos de crise os profissionais contam com outra estratégia que é o apoio da Polícia Militar (PM) e, tem a PM como parceira no atendimento das crises, diante do medo e do risco de agressividade que a equipe poderia sofrer, e ainda na necessidade de realizar a contenção física. As práticas dos profissionais do SAMU são pautadas na necessidade de segurança da equipe. ${ }^{16}$

A finalidade da existência da PM é responsabilizar-se pela segurança da sociedade, e essa função acabou de aproximar a polícia do louco, devido à construção social de que o doente mental possa oferecer periculosidade e atitudes imprevisíveis. ${ }^{13} 0$ estigma e 0 preconceito referente a doença mental foram construídos culturalmente, e do mesmo modo necessitam ser descontruídos. ${ }^{17}$

Conforme o protocolo do SAMU, o acionamento da PM, deve ser feito pela central de regulação médica, em casos que o usuário ofereça risco a cena, ${ }^{15}$ entretanto, os profissionais do serviço estudado também, utilizam a PM para realizar a contenção física, em casos em que 0 usuário se encontra agressivo. Embora, a PM seja vista pelos profissionais do SAMU como parceira durante os atendimentos, os próprios profissionais entendem que muitas vezes a contenção física pela polícia é compreendia de forma negativa pelos familiares dos usuários.

Os profissionais evidenciaram as medidas de contenção física e química como intervenções rotineiras, práticas predominantes adotadas pela psiquiatria tradicional, que visam solucionar as manifestações causadas pela crise e o controle do indivíduo. Estas práticas, são as mais representativas do modelo asilar, em razão de serem muito utilizadas nos manicômios, a fim de disciplinar o louco quando estes se mostram(vam) desobedientes, agressivos e resistentes aos tratamentos oferecidos. ${ }^{3}$

Dessa forma, é importante discutir a utilização destas práticas no modo da atenção psicossocial, pois o fato de utilizar essas medidas, não irá determinar se um modelo é manicomial ou não, mas a finalidade e o contexto em que esses métodos são utilizados. $^{13}$ A contenção física somente deverá ser usada em situações em que o usuário está em intensa agitação e com manifestação de comportamento agressivos, entretanto, apenas deve ser empregada quando as tentativas de intervenção verbal não forem suficientes para solucionar a crise. ${ }^{18}$

Em relação as medidas de contenção química, não se pode negar que o uso de psicofármacos podem ser um excelente recurso à disposição dos profissionais de saúde mental, porém o seu emprego de forma inadequada e excessiva, pode ser prejudicial, visto que na maioria das vezes, o uso indiscriminado de medicamentos psicotrópicos tem por objetivo a 


\section{JOURNAIOF \\ NURSING \\ ANo HEALTH}

ISSN 2236 - 1987

nos serviços como o SAMU, e isso exige um aumento de um cuidado que vise atender as necessidades dos usuários de forma cada vez mais resolutiva e humanizada. ${ }^{20}$

Um estudo internacional apontou que há muitos benefícios de priorizar as práticas não coercitivas, como redução de recursos, custos, prevenção de lesões, e melhores relações entre os profissionais e os usuários dos serviços de saúde mental. ${ }^{19} \mathrm{O}$ diálogo e a escuta ativa podem ser meios que permitem minimizar os sintomas agudos do transtorno mental como a ansiedade, os pensamentos suicidas, dentre outros. ${ }^{5}$

Os sujeitos deste estudo relataram que as intervenções oferecidas para a família se baseiam em uma orientação dos procedimentos a serem realizados com o seu familiar, como por exemplo, a contenção física e química, e a necessidade do encaminhamento do usuário para a atenção hospitalar.

Os profissionais do SAMU também encaminharam os familiares do usuário em crise para o serviço de assistência social dos pontos da RAPS, com o intuito de que tal serviço pudesse realizar $o$ acolhimento à família. Entende-se que, é importante que os profissionais do SAMU, busquem integrar os familiares aos cuidados de outros serviços da rede, contudo, os profissionais devem atentar para que a responsabilização do cuidado à família da pessoa em sofrimento psíquico não fique depositado somente no serviço social. É importante que o profissional que faz este encaminhamento incluase nesse processo, responsabilizando e 
ISSN $2236-1987$

compartilhando o caso, fazendo com que os serviços atuem em rede. ${ }^{6}$

Ressaltamos que os profissionais devem considerar a importância de acolher os familiares e/ou outras pessoas envolvidas na cena de atendimento, conforme o protocolo nacional do SAMU. ${ }^{15}$ É necessário que ao atender uma situação de crise psíquica, os profissionais considerem como a crise daquela família, daquele contexto social, pois a crise não afeta somente 0 sujeito em sofrimento psíquico, mas sim toda sua rede social e familiar. ${ }^{21}$ Uma crise psíquica geralmente tem um impacto emocional grande e duradouro para os usuários e seus familiares. ${ }^{11}$

A estratégia de atendimento oferecida à pessoa em situação de rua em crise psíquica, pelos profissionais do SAMU, tem como principal desfecho - encaminhamento para o hospital geral da região para dar continuidade à assistência ao usuário, e também com o intuito de que o serviço de assistência social do hospital geral de referência assuma a responsabilidade pelo caso da pessoa em situação de rua. Para o Ministério da Saúde, muitas vezes o SAMU tem dificuldade em atender 0 usuário com transtornos mentais em situação de rua, por considerar um problema exclusivamente social. 22

Os achados mostraram que na maioria dos atendimentos 0 direcionamento do usuário para outros pontos da RAPS, era para o hospital geral. Devido ao fato dos profissionais do SAMU terem a visão de que os serviços de base comunitária e territorial, não são efetivos para a continuidade do cuidado à crise. Estes achados corroboraram com outro estudo, ${ }^{23}$ no qual os profissionais demonstram desvalorização do funcionamento e resolutividade dos CAPS, em relação a sua função como um dos serviços substitutivos da RAPS ao modelo manicomial.

Dentre outros motivos destacados pela equipe e que também faz com que o hospital geral seja a referência para a continuidade do cuidado à crise, é não haver outros pontos da RAPS atuantes no munícipio para a atenção à crise no período noturno, finais de semana e feriados, a não ser o hospital geral. Nesse contexto, os profissionais do SAMU apontaram a necessidade de um CAPS do tipo III na região, para contemplar as necessidades dos usuários de saúde mental em tempo integral. Outro estudo realizado em um SAMU também evidenciou que a falta de um CAPS III torna a assistência fragmentada e pouco resolutiva. ${ }^{24} 0$ CAPS III proporciona ação continuada, com funcionamento 24 horas, incluindo finais de semana e feriados, ofertando retaguarda clínica e acolhimento noturno a outros serviços de saúde mental integrantes da RAPS. ${ }^{7}$

A ausência dos CAPS III nos municípios de pequeno porte populacional, contribui para fragilizar ações e a garantia de cuidados da crise no território. ${ }^{25} \mathrm{Em}$ um estudo, ${ }^{26}$ sobre o atendimento da crise nos diversos componentes da RAPS em Natal/RN, em que o município só dispõe de um CAPS III, os autores constaram que há leitos psiquiátricos insuficientes nos hospitais gerais e a inexistência dos CAPS tipo III em outras regiões da cidade do estudo, fazendo com que a população recorra ao hospital 
psiquiátrico, que ainda é o centro da rede no município estudado.

Houve um avanço na implantação dos serviços da RAPS no país, contudo, regiões de saúde com baixo desenvolvimento socioeconômico e de baixa oferta de serviços, há menor cobertura de CAPS III e CAPS AD III. ${ }^{27}$ Atualmente no Brasil, dados do Ministério da Saúde, ${ }^{28}$ informam que somente 3\% dos municípios brasileiros possuem CAPS III, e compreendemos que a ampliação da implantação deste serviço, seria um avanço para a Reforma Psiquiátrica brasileira e a saúde mental no país, já que em muitos lugares os CAPS II não conseguem sozinhos dar conta de toda a demanda de saúde mental do território.

\section{CONSIDERAÇÕES FINAIS}

Nesta pesquisa, identificou-se que as estratégias de atendimento dos profissionais que atuam no SAMU são fundamentadas nos saberes da psiquiatria clássica. 0 cuidado prestado pela equipe à pessoa com transtornos mentais em situação de crise, são centrados na contenção física e química, e não sendo o bastante, os profissionais têm a polícia militar como parceira durante 0 atendimento.

Uma limitação deste estudo refere-se à coleta de dados em relação à natureza do próprio serviço, que pelo fato de os profissionais estarem constantemente apreensivos ao receberem chamadas para atendimentos de emergência, em algumas entrevistas ficou evidente a necessidade das falam serem sintéticas e rápidas. Outra limitação enfrentada nesta pesquisa, diz respeito aos escassos estudos científicos encontrados tanto em contexto nacional quanto internacional que abordassem as estratégias de atendimento por serviços de emergência às situações de crise psíquica.

Diante disso, considera-se fundamental que as equipes que atuam no SAMU, estejam receptivas e motivadas para as atividades de educação permanente, baseadas nos cuidados preconizados pelo modo de atenção psicossocial e que novas concepções sobre o cuidado à crise psíquica tanto para o usuário quanto para a família, sejam apreendidos.

Percebeu-se no presente estudo que o hospital geral é referência pelos profissionais do SAMU para o encaminhamento do usuário em situação de crise psíquica, notando-se uma desvalorização referente aos serviços comunitários e territoriais para a continuidade do cuidado às crises psíquicas, e esta foi apontada como uma das principais dificuldades da equipe durante o atendimento.

Um avanço para a consolidação da RAPS no município estudado seria uma ação conjunta com os profissionais do CAPS, como foi referido pelos próprios participantes da pesquisa, podendo assim, prestar uma assistência de maior qualidade, bem como articulação das demandas de saúde mental com a atenção primária a saúde, já que, a mesma é responsável por organizar a rede e coordenar os cuidados.

$\mathrm{Na}$ presente pesquisa ficou evidente a necessidade da implantação 


\section{ISSN 2236 - 1987}

do CAPS III nos municípios de pequeno porte, oferecendo retaguarda clínica, e para que a responsabilidade da continuidade do cuidado à crise não seja depositada somente no hospital, esta seria outra medida de consolidação da RAPS no território estudado e para o avanço da Reforma Psiquiátrica brasileira.

\section{REFERÊNCIAS}

1 Brito AAC, Bonfada D, Guimarães J. Onde a reforma ainda não chegou: ecos da assistência às urgências psiquiátricas. Physis (Rio J.). [Internet]. 2015[acesso em 2019 mar 10];25(4):1293-312. Disponível em: http://www.scielo.br/pdf/physis/v25 n4/0103-7331-physis-25-04-01293.pdf

2 Almeida $A B$, Nascimento ERP, Rodrigues J, Zeferino MT, Souza AIJ, Hermida PMV. Mobile emergency medical services in the psychological crisis and the psychosocial paradigm. Texto \& contexto enferm. [Internet]. 2015[cited 2019 Mar. 10]; 24(4):103543. Available from: http://www.scielo.br/pdf/tce/v24n4/ 0104-0707-tce-24-04-01035.pdf

3 Willrich JQ. A crise psíquica no discurso da Atenção Psicossocial: um acontecimento de saber, poder e subjetivação [Tese] [Internet]. Pelotas (RS): Universidade Federal de Pelotas; 2016[acesso em 2019 abr 22\}]. Disponível em: https://wp.ufpel.edu.br/pgenfermage $\mathrm{m} /$ files/2017/03/TESE-JanaínaQuinzen-Willrich.pdf

4 Zeferino MT, Cartana MHF, Fialho MB, Huber MZ, Bertoncello KCG. Health workers' perception on crisis care in the psychosocial care network. Esc.
Anna Nery Rev. Enferm. [Internet]. 2016[cited 2019 Mar. 10];20(3):e20160059. Available from: http://www.scielo.br/pdf/ean/v20n3 /en_1414-8145-ean-20-03-

20160059.pdf

5 Alcântara CB, Capistrano FC, Czarnobay J, Ferreira ACZ, Brusamarello T, Maftum MA. Drug therapy for people with mental disorders in the view of nursing professionals. Esc. Anna Nery Rev. Enferm. [Internet]. 2018[cited 2019 Apr. 12]; 22(2);e20170294. Available from:

http: / /www.scielo.br/pdf/ean/v22n2 /pt_1414-8145-ean-2177-9465-EAN2017-0294.pdf

6 Andrade K, Zeferino MT, Fialho, MB. Articulação da rede de atenção psicossocial para o cuidado às crises. Psicol. estud. [Internet]. 2016[acesso em 2019 abr 12]; 21(2):223-33. Disponível em: https: / /www.redalyc.org/articulo.oa? id $=287147424005$

7 Ministério da Saúde (BR). Portaria $\mathrm{n}^{\circ}$ 3.088, de 23 de dezembro de 2011: institui a Rede de Atenção Psicossocial para pessoas com sofrimento ou transtorno mental e com necessidades decorrentes do uso de crack, álcool e outras drogas, no âmbito do Sistema Único de Saúde [Internet]. Brasília; 2011[acesso em 2019 abr 22]. Disponível em: http: / /bvsms.saude.gov.br/bvs/saude legis/gm/2011/prt3088_23_12_2011_r ep.html

8 Ministério da Saúde (BR). Secretaria de Atenção à Saúde. Saúde Mental: cadernos de atenção básica $n^{\circ} 34$ [Internet]. Brasília; 2013[acesso em 


\section{ISSN 2236 - 1987}

2019 abr 22]. Disponível em: http://189.28.128.100/dab/docs/port aldab/publicacoes/caderno_34.pdf

9 Souza AS, Cortes HM, Pinho PH. Serviços de atendimento móvel de urgência frente às emergências psiquiátricas: Uma revisão narrativa. Rev. port. enferm. saúde mental. [Internet]. 2018[acesso em 2019 abr 12];(20):72-80. Disponível em: http://www.scielo.mec.pt/scielo.php ?script=sci_abstract\&pid=S1647-

$21602018000300010 \& \operatorname{lng}=$ en\& $\mathrm{nrm}=$ iso \&ttlng=pt

10 Campos PJ. A rede de atenção psicossocial (raps) e os pontos estratégicos na atenção à crise e urgência. In: Zeferino MT, Rodrigues J, Assis JT, organizadores. Crise e urgência em saúde mental: organização da atenção psicossocial à crise e o cuidado em rede. $4^{\text {a }}$ ed. Florianópolis: Universidade Federal de Santa Catarina; 2015: 11-36.

11 Daggenvoorde TH, Gijsman HJ, Goossens PJJ. Emergency care in case of acute psychotic and/or manic symptoms: lived experiences of patients and their families with the first interventions of a mobile crisis team: a phenomenological study. Perspect Psychiatr Care [Internet]. 2017[cited 2019 Mar. 10]; 54:462-8. Available from: https://onlinelibrary.wiley.com/doi/e pdf/10.1111/ppc.12247

12 Bastos FBS, Dutra CDC, Silva JLA, Pacheco KCV, Silva TN. Saúde mental no atendimento pré-hospitalar móvel: Concepções de profissionais. Rev. port. enferm. saúde mental [Internet]. 2016[acesso em 2019 abr 12];4(n.esp.):17-24. Disponível em: http://www.scielo.mec.pt/scielo.php ?script=sci_arttext\&pid=S164721602016000400003

13 Willrich JQ, Kantorski LP, Antonacci $\mathrm{MH}$, Cortes JM, Chiavagatti FG. Da violência ao vínculo: construindo novos sentidos para a atenção à crise. Rev. bras. enferm. [Internet]. 2014[acesso em 2019 mar 10];67(1):97-103. Disponível em: http://www.scielo.br/pdf/reben/v67 n1/0034-7167-reben-67-01-0097.pdf

14 Minayo MCS. Pesquisa Social: teoria, método e criatividade. Petrópolis: Vozes; 2016.

15 Ministério da Saúde (BR). Secretaria de Atenção à Saúde. Protocolo de Intervenção para o SAMU 192 [Internet]. $2^{\mathrm{a}}$ ed. Brasília; 2016[acesso em 2019 abr 22]. Disponível em: http://portalarquivos.saude.gov. $\mathrm{br} / \mathrm{i}$ mages/pdf/2016/outubro/26/livroavancado-2016.pdf

16 Almeida $A B$, Nascimento ERP, Rodrigues J, Schweitzer G. Intervenção nas situações de crise psíquica: dificuldades e sugestões de uma equipe de atenção pré-hospitalar. Rev. bras. enferm. [Internet]. 2014[acesso em 2019 mar 10];67(5): 708-14. Disponível em: http://www.scielo.br/pdf/reben/v67 n5/0034-7167-reben-67-05-0708.pdf

17 Cortes HM, Barros S. Reabilitação psicossocial de moradores de um serviço residencial terapêutico. J. nurs. health. [Internet]. 2017[acesso em 2019 abr 15]; 7(2):148-63. Disponível em: https://periodicos.ufpel.edu.br/ojs2/ index.php/enfermagem/article/view/ $12313 / 7885$ 


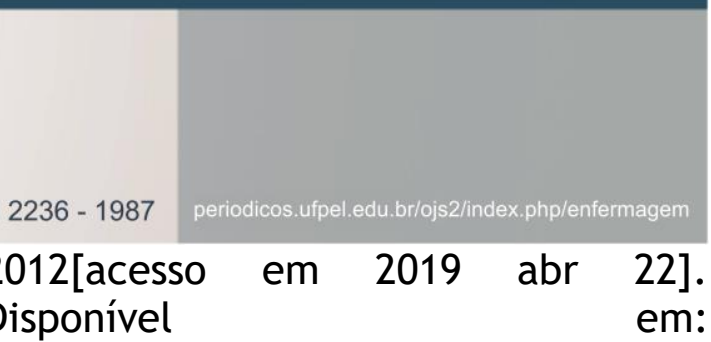

18 Santos ACT, Nascimento YCML, Lucena TS, Rodrigues PM da S, Brêda MZ, Santos GF. Mobile service attendance of urgency to psychiatric urgencies and emergencies. Rev. enferm. UFPE on line. [Internet]. 2014[cited 2019 mar 10]; 8(6): 158696. Available from: https: / / periodicos.ufpe.br/revistas/ $r$ evistaenfermagem/article/view/9849 /10060

19 Zeller SL, Citrome L. Managing agitation associated with schizophrenia and bipolar disorder in the emergency setting. West $\mathrm{J}$ Emerg Med [Internet]. 2016[cited 2019 mar 10];17(2):165-72. Available from: https://www.ncbi.nlm.nih.gov/pmc/a rticles/PMC4786236/pdf/wjem-17165.pdf

20 Oliveira LC, Silva RAR. Saberes e práticas em urgências e emergências psiquiátricas. Rev. enferm. UERJ. [Internet]. 2017[acesso em 2019 abr 12]; 25:e10726. Disponível em: https: / /www.e-

publicacoes.uerj.br/index.php/enfer magemuerj/article/view/10726

21 Fialho MB. Atenção à crise e urgência em saúde mental. Contexto histórico, epistemológico e social. Diferença entre crise e urgência. In: Zeferino MT; Rodrigues J, Assis JT, organizadores. Crise e urgência em saúde mental: o cuidado às pessoas em situações de crise e urgência na perspectiva da atenção psicossocial. $4^{\text {a }}$ ed. Florianópolis: Universidade Federal de Santa Catarina; 2015:11-39.

22 Ministério da Saúde (BR). Secretaria de Atenção à Saúde. Manual sobre o cuidado à saúde junto à população em situação de rua [Internet]. Brasília;
ISSN 2236 - 1987

Disponível em:
http://189.28.128.100/dab/docs/publ icacoes/geral/manual_cuidado_popul alcao_rua.pdf

23 Bonfada D, Guimarães J, Miranda FAN, Brito AAC. Reforma psiquiátrica brasileira: conhecimentos dos profissionais de saúde do serviço de atendimento móvel de urgência. Esc. Anna Nery Rev. Enferm. [Internet]. 2013[acesso em 2019 mar 10];17(2);227-33. Disponível em: http://www.scielo.br/pdf/ean/v17n2 /v17n2a04.pdf

24 Oliveira LC, Silva RAR, Carvalho FPB, Soares FRR, Sousa KMN, Solano LC. Dificuldades no atendimento às urgências e emergências psiquiátricas no serviço de atendimento móvel de urgência. Enferm. foco (Brasília). [Internet]. 2018[acesso em 2019 abr 15];9(4):18-22. Disponível em: http://revista.cofen.gov.br/index.php /enfermagem/article/view/1317/471

25 Macedo JP, Abreu MM, Fontenele MG, Dimenstein M. A regionalização da saúde mental e os novos desafios da Reforma Psiquiátrica brasileira. Saúde Soc. [Internet]. 2017[acesso em 2019 mar 10];26(1):155-70. Disponível em: http://www.scielo.br/pdf/sausoc/v26 n1/1984-0470-sausoc-26-01-00155.pdf

26 Dimenstein $M$, Amorim AKA, Leite J, Siqueira K, Gruska V, Vieira C et al. O atendimento da crise nos diversos componentes da rede de atenção psicossocial em Natal/RN. Rev. polis psique. [Internet]. 2012[acesso em 2019 mar 10];2(3):95-27. Disponível em:

https: / / seer.ufrgs.br/PolisePsique/art icle/view/40323/25630 
27 Dimenstein M, Macedo JP, Gomes M, Silva TM, Abreu MM. A saúde mental e atenção psicossocial: regionalização e gestão do cuidado integral no SUS. Salud soc. [Internet]. 2018[acesso em 2019 mar 15];9(1):70-85. Disponível em:

https: / / dialnet.unirioja.es/servlet/ar ticulo? codigo $=6755334$

28 Ministério da saúde (BR). Saúde mental no SUS cuidado em liberdade, defesa de direitos e rede de atenção psicossocial: relatório de gestão 20112015 [Internet]. Brasília; 2016[acesso em 2019 abr 22]. Disponível em: http://portalarquivos2. saude.gov.br/i mages/pdf/2016/junho/27/Relat--rioGest--o-2011-2015---.pdf

Data de submissão: 24/02/2019

Data de aceite: 14/04/2019

Data de publicação: 24/04/2019 